\title{
PERSISTENCIA DEL CONDUCTO ARTERIOSO CON FLUJO DERECHA-IZQUIERDA EN CANINOS: DISCUSIÓN DE UN CASO
}

Bezold Arnillas, Ursula ${ }^{1}$

Del Valle Ballón, Paola ${ }^{2}$

\section{RESUMEN:}

El objetivo del presente artículo es hacer una revisión de un caso clínico de Conducto Arterioso Persistente de flujo derechaizquierda, también conocido como Conducto Arterioso Persistente Reverso (CAP reverso) en un canino de raza Scottish Terrier, que se diagnosticó en Lima en mayo del 2008. Mediante este artículo se busca destacar la sintomatología observada, los métodos que condujeron al diagnóstico, así como el seguimiento que se hizo al paciente hasta su fallecimiento.

Palabras clave: Conducto Arterioso Persistente, CAP reverso, cianosis diferencial.

\begin{abstract}
:
The purpose of this article is to review the clinical case of a Persistent Arterial Duct with flow right to left, also known as Reversed Patent Ductus Arteriosus (PDA reversed) in a Scottish Terrier dog, which was diagnosed in Lima in May, 2008. This article is focused to emphasize the observed clinical symptoms, the methods that led to the diagnosis, as well as the follow-up done to the patient up to death.
\end{abstract}

Key words: Persistent Arterial Duct right to left, PDA reversed, differential cyanosis.

1 Cátedras de Cirugía de Animales Menores y Principios Básicos de Cardiología Veterinaria, EAP Ciencias Veterinarias - Facultad de Ciencias Biológicas, Universidad Ricardo Palma. Email: dra_ub@yahoo.com

2 Práctica privada. Clínica Veterinaria SOS 


\section{INTRODUCCIÓN:}

La persistencia del conducto arterioso o Conducto Arterioso Persistente (CAP) es el defecto cardiaco congénito con mayor incidencia de presentación en caninos, siendo más frecuente en hembras que machos. Se ha demostrado como rasgo multigenético en diversas razas caninas como: Chihuahua, Collie, Maltés, Poodle, Pomerania, Pastor de Shetland, Bichon Frisé, Cocker Spaniel, Pastor Alemán, Springer Spaniel Inglés, Labrador Retriever, Yorkshire Terrier y Terranova. (Kittleson y Kienle, 1998; Ramirez, 2009).

Durante la vida fetal, el conducto arterioso sirve para desviar la mayor parte del caudal de sangre oxigenada proveniente de la madre directamente hacia la arteria aorta del feto, evitando a los pulmones de éste ya que todavía no son funcionales. El cierre del conducto arterioso debería ocurrir normalmente a pocas horas del nacimiento, y es seguido por cambios estructurales que ocurren en las siguientes semanas, lo que causa un cierre permanente. Se considera anormal si el conducto arterioso permanece abierto por más de los 7 a los 10 días de nacido.

Cuando el conducto falla en cerrarse, usualmente la sangre se desvía desde la aorta hacia la arteria pulmonar (CAP de izquierda-derecha), debido a la mayor presión a nivel de la aorta que en la pulmonar, ocurriendo flujo tanto en sístole como en diástole, lo cual se traduce en la auscultación de un soplo continuo o de maquinaria. Como consecuencia se va a incrementar el flujo pulmonar y por ende el retorno venoso por medio de las venas pulmonares hacia el lado izquierdo del corazón, desarrollando dilatación atrial y ventricular izquierda, hipertrofia excéntrica e incremento de la presión al final de la diástole. Si el defecto es muy amplio para permitir una mayor comunicación y que la resistencia vascular pulmonar permanezca baja, el resultado final puede ser la falla ventricular con edema pulmonar (Ramirez, 2009).

Cuando la resistencia vascular pulmonar aumenta y rebasa la resistencia vascular sistémica, la sangre se va a desviar de la arteria pulmonar a la aorta, lo cual se denomina Conducto Arterioso Persistente de flujo 
derecha- izquierda o CAP reverso. La literatura menciona que el $15 \%$ de los perros que han heredado una persistencia del conducto arterioso desarrollan un CAP reverso. Estos pacientes van a presentar una marcada hipertrofia del ventrículo derecho; las presiones en la arteria pulmonar y en la aorta tienden a ser similares, y no se auscultará soplo continuo en maquinaria. En ocasiones puede auscultarse un desdoblamiento del segundo ruido cardiaco. (Ramirez, 2009).

Kittleson y Kienle (1998) mencionan que la mayoría de perros con CAP reverso presentan un conducto arterioso grande que no opone resistencia al flujo sanguíneo. La falta completa de cierre produce una comunicación entre la aorta y la arteria pulmonar del mismo tamaño que estos grandes vasos, de tal manera que la resistencia al flujo entre estos dos vasos es inexistente o muy pequeña. En consecuencia, la sangre fluye desde el lado derecho e izquierdo del corazón por estos lechos vasculares en cantidad proporcional a la resistencia al flujo de cada uno de estos lechos vasculares y las presiones en la aorta y la arteria pulmonar se equilibran. Bajo esta situación, cabe esperar que el flujo sanguíneo pulmonar sea aproximadamente 5 veces mayor que el flujo sistémico porque la resistencia vascular pulmonar es generalmente una quinta parte de la resistencia vascular sistémica. Cuando la comunicación es clínicamente significativa, gran cantidad de sangre desoxigenada pasa de la circulación venosa (arteria pulmonar) a la aorta. Así, se produce una disminución de la presión de oxígeno arterial más allá de la región en la que el conducto se une con la aorta, en reposo o bien durante el ejercicio; el ejercicio produce un aumento de la comunicación derecha-izquierda y agrava la cianosis.

La CAP reversa disminuye la oxigenación renal con el consiguiente aumento de la liberación de eritropoyetina, lo que produce un aumento de la eritropoyesis que deriva en policitemia (aumento del hematocrito). La policitemia compensa la disminución de los efectos de la hipoxia a nivel tisular, siendo un efecto beneficioso cuando el hematocrito es de 55-65\%. Sin embargo, la policitemia se torna perjudicial cuando el hematocrito aumenta hasta el punto de que la sangre se torna hiperviscosa, lo cual suele suceder cuando el hematocrito llega a valores de 70-75\%. El aumento 
de la viscosidad aumenta la resistencia al flujo sanguíneo, alterándose la irrigación sistémica lo que disminuye el aporte de oxígeno a los tejidos. (Kittleson y Kienle, 1998; Mucha et al. 2004; Tilley et al. 2008).

\section{DESCRIPCIÓN DEL CASO CLÍNICO}

Se presentó a consulta un canino macho, de raza Scottish Terrier, de ocho meses de edad y $8 \mathrm{~kg}$. de peso. El motivo de la consulta fue que la propietaria lo observó decaído al día siguiente de haberlo visto mordiendo una pantufla.

Al examen físico manifestó dolor abdominal a la palpación; las demás constantes fisiológicas se encontraron dentro de los rangos normales, sospechándose de ingesta de material extraño (esponja o relleno de la pantufla). Suponiendo obstrucción por cuerpo extraño se le realizó un examen radiológico de tránsito baritado, descartándose la obstrucción, aunque quedaron finas trazas de bario a nivel gástrico e intestinal, asumiéndose que se trataba de lesiones en la mucosa debido al paso del cuerpo extraño.

Se practicó un hemograma, el cual arrojó un hematocrito de $76 \%$ y hemoglobina de $27 \mathrm{~g} / \mathrm{dl}$, siendo los demás normales. Se administró terapia de fluidos endovenosos junto a un protector hepático y ranitidina; luego de unas horas en observación, el paciente se retiró de la clínica bastante mejor.

A los dos días regresó el paciente para su control y se observó bastante vivaz; sin embargo, tras la manipulación del examen físico vomitó restos de alimento con pelos, por lo que se asumió que seguía ingiriendo todo tipo de cosas. Se aumentó sucralfato a la receta y se le recomendó dieta blanda, además de vigilar que no ingiriera materiales extraños. Se le practicó un nuevo hemograma y el hematocrito estaba en $71 \%$ y la hemoglobina en $24 \mathrm{~g} / \mathrm{dl}$, por lo que se le administró más líquidos endovenosos (a pesar que el animal aparte del vómito observado, estaba bastante bien). También se realizó una ecografía abdominal que no evidenció ninguna anormalidad a excepción de una vesícula biliar ligeramente dilatada. El propietario reportó los días siguientes que el animal había vuelto a la normalidad. 
Una semana después de la última visita lo llevaron de emergencia porque el animal presentaba temblores y se quejaba. En la exploración se detectó mucho dolor abdominal y temperatura rectal de $40^{\circ} \mathrm{C}$, siendo la primera vez que se le detectaba fiebre. No habían vómitos ni diarreas. Se le administró flunixin-meglumine, observando mejoría. A día siguiente se realizó un nuevo hemograma, obteniendo un hematocrito de $80 \%$. A este punto se sospechó que el problema era más serio que una simple indigestión por comer materiales extraños, por lo que se decidió realizar una laparotomía exploratoria.

Durante la laparotomía exploratoria se observaron los intestinos cianóticos, vasos mesentéricos en apariencia congestivos, y el bazo estaba agrandado y de un color muy oscuro. Todos los vasos mesentéricos estaban en apariencia congestionados; recién pudo observarse pulsaciones a nivel de las arterias mesentéricas a raíz del manipuleo de las asas intestinales en busca de algún contenido (se palpó desde estómago hasta el colon sin detectar cuerpos extraños, pero fue evidente que el manipuleo favorecía la circulación de estos órganos los cuales mejoraron su apariencia sin llegar a ser completamente rosados). Se evaluó detenidamente toda la zona hepático-portal en busca de algún shunt o puente vascular extrahepático anormal, pero no se encontró. Tampoco se observaron signos de peritonitis.

En repetidas ocasiones durante la laparotomía se llamó la atención al anestesista ante la evidencia de cianosis en el campo operatorio; sin embargo, el anestesista repetidamente manifestó que la pulsioximetría era normal (el sensor estaba colocado en la lengua del paciente) y pudo comprobarse el color rojizo de las mucosas orales, así como un adecuado llenado capilar. Al finalizar el cierre de la cavidad abdominal se examinó la mucosa peneana, la cual estaba cianótica, a diferencia de las mucosas orales y conjuntivales que tenían una coloración normal.

Con todos estos datos se determinó que el problema primario era la POLICITEMIA, tratándose de una policitemia absoluta (masa eritroide incrementada asociada a una mayor producción en la médula ósea). Como los riñones estaban bien en apariencia y la bioquímica estaba dentro de los rangos se descartó el origen renal del exceso de eritropoyetina. Así se llegó a dos posibles diagnósticos: Policitemia Vera o Policitemia secundaria a pobre oxigenación debida a algún defecto vascular congénito. 
Cabe mencionar que después de la cirugía la propietaria refirió que este perro era bastante tranquilo a diferencia de su hermano de camada que era bastante hiperactivo. Externamente no había diferencias en cuanto al desarrollo corporal y tamaño de ambos animales.

Se decidió practicar una flebotomía, extrayendo alrededor de $100 \mathrm{ml}$ de sangre, la cual fue reemplazada por suero fisiológico EV. El paciente tuvo una recuperación postquirúrgica bastante buena, a pesar que la policitemia persistía: el hematocrito a los 2 días de la cirugía era de $61 \%$ y a los 5 días era de $64 \%$.

Teniendo en cuenta todos estos datos, más la evidencia de un tipo particular de cianosis exclusiva de las vísceras abdominales y el tren posterior observada durante la laparatomía ("cianosis diferencial") y que persistió en los días siguientes a la cirugía, se sospechó de un Conducto Arterioso Persistente derecha-izquierda (CAP reverso)

Para confirmar el diagnóstico se procedió a realizar la evaluación ecocardiográfica. Es importante señalar que en esa época (mayo 2008) no se contaba en Perú con Ecocardiografía Doppler color para uso veterinario (Dávila y Martínez, comunicación personal), por lo que en un primer momento se realizó la evaluación clásica en modos $\mathrm{B}$ y $\mathrm{M}$, obteniendo las medidas sistólicas: pared libre del ventrículo derecho $1.24 \mathrm{~cm}$, tabique interventricular $1 \mathrm{~cm}$, y pared libre del ventrículo izquierdo de $1.04 \mathrm{~cm}$; con lo cual resultó obvia la hipertrofia ventricular derecha. Para visualizar el flujo sanguíneo sin contar con sistema doppler se decidió hacer una prueba ecográfica mencionada por la literatura, consistente en utilizar suero salino con microburbujas como contraste, el cual inyectamos a través de la canulación de la vena cefálica al mismo tiempo que se ecografió el corazón a través de la ventana paraesternal derecha, observando el pasaje de las burbujas solo en las cavidades derechas del corazón (descartando comunicación intracardiaca de flujo derecha-izquierda). Inmediatamente después se repitió la prueba pero ecografiando la aorta abdominal, en la cual sí pudimos detectar el pasaje de las burbujas (sin pasar por el ventrículo izquierdo), por lo que se dedujo que las burbujas pasaron del ventrículo derecho y la arteria pulmonar directamente a la aorta sin pasar previamente por el lado izquierdo del corazón, con lo cual se confirmó la presencia del CAP reverso. 
No se realizaron radiografías ya que la propietaria pidió priorizar los exámenes que más datos aportaran por motivos de costos. Ya confirmada la cardiopatía congénita (CAP reverso) se descartó la posibilidad de cirugía ya que esta agravaría mortalmente la hipertensión pulmonar, optándose por un tratamiento a base de flebotomías e hidroxiúrea. Adicionalmente se planteó la posibilidad del empleo de ácido acetilsalicílico solo en caso de incremento del conteo de plaquetas, así como el uso de atenolol si es que se presentaran arritmias.

Una vez que el paciente se recuperó de la cirugía se programaron hemogramas cada 15 días. La idea fue mantener un hematocrito entre 55 y $65 \%$, que permitieran un flujo menos viscoso de la sangre pero que al mismo tiempo compense en cierto grado la cianosis de la mitad caudal del organismo del paciente. En los casos en que el hemograma resultara mayor a $65 \%$ habría que realizar flebotomías, reemplazando el volumen sanguíneo extraído por suero fisiológico endovenoso. Durante los primeros 7 meses el paciente se mostró estable, siendo necesarias las flebotomías en promedio cada 6 semanas. En los primeros meses del 2009 las flebotomías fueron en promedio una al mes, pero a partir de mayo de ese año se observó que el hematocrito tendía a mantenerse estable entre 55$65 \%$. A inicios del mes de agosto de ese mismo año el paciente fue traído a consulta por mostrar letargia y aparente debilidad. Se tomaron muestras para hemograma y bioquímica sanguínea, obteniendo un hematocrito de $51 \%$, hemoglobina de $16,9 \mathrm{~g} / \mathrm{dl}$, creatinina de $3,06 \mathrm{mg} / \mathrm{dl}$ y úrea de 301 $\mathrm{mg} / \mathrm{dl}$, diagnosticándose falla renal. Los días siguientes se procedió a la terapia de fluidos para neutralizar la uremia, repitiéndose los análisis 6 días después, obteniendo un hematocrito de 49,4\%, hemoglobina de16,6 $\mathrm{g} / \mathrm{dl}$, creatinina de $3,47 \mathrm{mg} / \mathrm{dl}$ y úrea de $228 \mathrm{mg} / \mathrm{dl}$. Si bien la uremia había disminuido ligeramente, la falla renal persistía, con el consecuente descenso en los niveles de eritropoyetina que se traducía en un hematocrito menor. Se continuó con el tratamiento pero 10 días después los análisis mostraron un hematocrito de 51,3\%, hemoglobina de $16,8 \mathrm{~g} / \mathrm{dl}$, creatinina de $4,65 \mathrm{mg} / \mathrm{dl}$ y úrea de $277 \mathrm{mg} / \mathrm{dl}$, revelando la continuidad del deterioro renal. Una semana después del último análisis el paciente fue llevado de emergencia por encontrarse sumamente debilitado, procediéndose a la eutanasia a solicitud de su propietaria. 


\section{DISCUSIÓN}

El diagnóstico de CAP reverso es un evento raro. La literatura menciona por ejemplo un estudio realizado en 215 perros entre 1986 y 1996 en la Universidad de California, de los cuales solo 14 presentaron CAP derecha-izquierda. Estos catorce perros pertenecían a las razas: Poodle miniatura, Cocker Spaniel, Springer Spaniel Inglés, Pastor de Shetland, Pointer, Galés de Cardigan, Siberian Husky, Pastor Australiano, Maltés y Labrador Retrieves. (Kittleson y Kienle, 1998).

En cuanto a los hallazgos clínicos, la literatura menciona que el problema que más reportan los propietarios es que el animal cae del tercio posterior durante el ejercicio, signo clínico que no fue comunicado por la propietaria de nuestro paciente. El dolor abdominal (cólico) propiamente dicho no es mencionado por la literatura, aunque si se hace referencia a dolor específico o inespecífico (Kittleson y Kienle, 1998; Ribeiro 2007). Otros signos clínicos reportados son: letargia, anorexia y signos neurológicos como convulsiones, colapso y síncope (Kittleson y Kienle 1998; Ramírez 2009). Estos signos clínicos estarían asociados a la policitemia que ocasiona hiperviscosidad y enlentecimiento del flujo en los vasos sanguíneos de pequieño calibre (Kittleson y Kienle 1998; Ribeiro 2007).

Otro signo clave es la cianosis diferencial, la que sí se observó en nuestro paciente, la cual es visible tanto en reposo como después del ejercicio. Esta se debe a que la sangre no oxigenada ingresa a la aorta después del nacimiento del tronco braquiocefálico y la arteria subclavia izquierda (vasos de la cabeza), y se dirige hacia la parte caudal del organismo. De esta forma, la cabeza y las extremidades anteriores reciben sangre oxigenada, y la región caudal del organismo recibe sangre desoxigenada. El resultado es un color normal de la mucosa oral y un color cianótico de las mucosas del prepucio, pene y la vulva. (Kittleson y Kienle 1998; Ribeiro 2007).

Otro hallazgo clínico que reporta la literatura es la auscultación de desdoblamiento del segundo ruido cardiaco (Kittleson y Kienle 1998; Ribeiro 2007; Ramírez 2009). Los CAP derecha-izquierda no acostumbran presentar soplos (Kittleson y Kienle 1998). 
En cuanto a los hallazgos de laboratorio, la literatura menciona que en las comunicaciones de derecha-izquierda comúnmente el hematocrito está elevado por encima de 55\% (Ribeiro 2007), lo cual coincide con los elevados hematocrito de nuestro paciente.

La ecocardiografía se considera el examen complementario más importante en casos de malformaciones cardiovasculares congénitas, recomendando el sistema Doppler color para identificar el CAP, observándose el flujo desde la aorta hacia la arteria pulmonar (Ribeiro 2007; Ramírez 2009). Ante la imposibilidad de utilizar el Doppler color, la literatura menciona la ecocardiografía de contraste, la cual consiste en inyectar suero fisiológico con microburbujas en una vena periférica mientras se realiza el estudio de la imagen cardiaca descartando inicialmente una comunicación derecha-izquierda intracardiaca. Hecho este primer descarte, se asume que existe una comunicación extracardiaca, la cual se confirma mediante la visualización de las microburbujas a nivel de la aorta abdominal (Kittleson y Kienle 1998; Kienle y Thomas 2004; Ramírez 2009).

Otros hallazgos ecocardiográficos son: dilatación de atrio derecho, hipertrofia del ventrículo derecho, insuficiencia tricuspídea, cámaras izquierdas normales o disminuidas, dilatación de la arteria pulmonar principal (Kienle y Thomas 2004; Ramírez 2009).

Con respecto al tratamiento, este difiere de lo recomendado para los CAP izquierda-derecha donde la cirugía de cierre del conducto tiene un excelente pronóstico. En los CAP reversos la cirugía está contraindicada ya que produciría hipertensión pulmonar mortal, ya que en estos casos el conducto funciona como una válvula de escape para el ventrículo derecho (Ramírez 2009). La literatura menciona que en los casos que se realizó cirugía, la muerte del paciente se produjo dentro de las 36 horas tras la cirugía (Kittleson y Kienle 1998).

El tratamiento de los perros con comunicación derecha-izquierda va dirigido a disminuir el hematocrito. La mayoría de los síntomas observados en perros con CAP reverso son consecuencia de la hiperviscosidad debida a la policitemia. En consecuencia, si se trata la policitemia es posible controlar a los pacientes a largo plazo. El procedimiento de elección es 
la flebotomía. Una disminución pequeña del hematocrito se acompaña de una mejoría clínica significativa, mientras que una flebotomía excesiva podría disminuir el aporte de oxígeno a los tejidos a niveles críticos. En general, se busca disminuir el hematocrito a $60-65 \%$. (Kittleson y Kienle, 1998).

En los casos en los que es necesario realizar flebotomías frecuentes o que no toleran la flebotomía, puede administrarse hidroxiúrea. La hidroxiúrea es un fármaco mielodepresor que produce inhibición transitoria de la médula ósea. Es necesario realizar hemogramas y recuentos plaquetarios de control cada 1-2 semanas. Si aparecen leucopenia, trombocitopenia o anemia debe interrumpirse el tratamiento hasta que se normalicen los valores. Posteriormente, es posible administrar una dosis más baja. Los efectos secundarios de la hidroxiurea en el perro incluyen anorexia, vómitos, hipoplasia de médula ósea y desprendimiento de las uñas. (Kittleson y Kienle, 1998)

La falla renal, que fue la causa del deterioro final de nuestro paciente y que motivó la eutanasia, se asocia a la pobre oxigenación renal, que si bien en un primer momento generó el incremento de la producción de eritropoyetina, a largo plazo produjo el deterioro prematuro de la corteza renal, ocasionando la uremia observada. 


\section{REFERENCIAS BIBLIOGRÁFICAS}

Kienle R, Thomas W. 2004. Ecocardiografía. En: Nyland, T. Diagnóstico ecográfico en pequeños animales. $2^{\circ}$ edición. Barcelona; Multimédica Ediciones Veterinarias, p 406-409.

Kittleson M, Kienle R. 1998. Medicina cardiovascular de pequeños animales. $2^{\circ}$ edición. Barcelona; Ed. Mosby. 600 p.

Mucha C, Sorribas C, Pellegrino F. 2005. Consulta rápida en la clínica diaria. $1^{\circ}$ edición. Buenos Aires; Intermédica. 770 p.

Ramírez A. 2009. Enfermedades cardiacas congénitas en perros y gatos. En: Mucha, J. Diplomado a distancia: Cardiología en perros y gatos. Módulo V. México; Centro de Estudios Avanzados en Medicina Veterinaria, $\mathrm{p} 19-30$

Ribeiro A. 2007. Conducto arterioso persiste (CAP). En: Belerenian, G; Mucha, C; Camacho, A; Manubens, J. Afecciones cardiovasculares en pequeños animales. $2^{\circ}$ edición. Buenos Aires; Ed. Inter-Médica, p 207-213.

Tilley L, Smith F, Oyama M, Sleeper M. 2008. Manual of canine and feline cardiology. $4^{\circ}$ edición. USA; Saunders. 464 p. 